\title{
Carnets
}

Revue électronique d'études françaises de l'APEF

Deuxième série - $12 \mid 2018$

Théorie Mimétique et Études Littéraires

\section{Wordsworth, Girard et la question de la propriété intellectuelle}

\section{Antonio Machuco Rosa}

\section{(2) OpenEdition}

Journals

Édition électronique

URL : http://journals.openedition.org/carnets/2511

DOI : 10.4000/carnets.2511

ISSN : 1646-7698

Éditeur

APEF

Référence électronique

Antonio Machuco Rosa, « Wordsworth, Girard et la question de la propriété intellectuelle », Carnets [En ligne], Deuxième série - 12 | 2018, mis en ligne le 19 janvier 2018, consulté le 19 avril 2019. URL : http://journals.openedition.org/carnets/2511; DOI : 10.4000/carnets.2511

Ce document a été généré automatiquement le 19 avril 2019.

\section{(c) (i) (8)}

Carnets est mis à disposition selon les termes de la licence Creative Commons - Atribution - Pas d'utilisation commerciale 4.0 International. 


\title{
Wordsworth, Girard et la question de la propriété intellectuelle
}

\author{
Antonio Machuco Rosa
}

1 En 1802 William Wordsworth publie la Préface à la seconde édition de ses Ballades Lyriques, un texte considéré, notamment en Angleterre, comme un des manifestes fondateurs de l'esthétique romantique. Y figure une déclaration bien connue de Wordsworth sur la nature de la poésie :

(...) poetry is the spontaneous overflow of powerful feelings: it takes its origin from emotion recollected in tranquility: the emotion is contemplated till, by a species of reaction, the tranquility gradually disappears, and an emotion, kindred to that which was before the subject of contemplation, is gradually produced, and does itself actually exist in the mind (Wordsworth, 1974, 1: 149).

2 Le poète éprouve des sentiments intenses dans la tranquillité de son retrait. Ce sont des sentiments spontanés qui causent une émotion dont l'origine se trouve dans l'être individuel $\mathrm{du}$ poète et non pas en une quelconque imitation d'autres individus. Ils manifestent l'identité singulière de l'auteur, laquelle est la source de la création d'une oeuvre originale donnée au lecteur. Dans la mesure où il exprime des sentiments originaux, le poète possède une sensibilité supérieure et une singularité unique qui le distingue du commun des mortels. Il s'adresse à d'autres individus mais s'écarte d'eux parce qu'«il pense et sent rapidement sans une influence externe immédiate et parce qu'il a une plus grande capacité d'exprimer ces pensées et sentiments qui jaillissent en lui en absence de cette influence " (Wordsworth, 1974, $1: 142$ ). Certes, Wordsworth affirme que le poète n'est pas influencé par l'extérieur et ne parle que de par sa personne et son caractère, mais il est vrai également que, dans la Préface de 1802, la supériorité du poète face aux autres hommes est relativisée. Bien que le poète ne soit pas guidé par l'imitation, les passions et les sentiments qu'il exprime sont les passions et les sentiments éprouvés par les autres hommes, la seule différence consistant dans leur intensité qui est plus élevée dans le cas du poète ; il ne s'agit donc pas d'une différence de nature mais de degré (Ibid.). Le poète partage le langage des autres hommes et n'écrit pas seulement pour l'élite des Poètes mais aussi pour l'homme commun. Il doit donc « descendre de son pinacle et, à fin de provoquer une simpathie rationnelle, il a à s'exprimer comme les autres hommes 
s'expriment " (Wordsworth, 1974, 1: 143). Le poète se doit de sortir de sa position supérieure et de chercher le lecteur, et c'est la nécessité même de ce mouvement qui constitue le sujet central de la Préface des Ballades Lyriques. Donc, dans le texte de 1802, Wordsworth n'a fait que briser en partie avec la conception classique de l'art selon laquelle celui-ci doit aller vers l'audience et lui plaire (cf., par exemple, Abrams, 1953). En effet, «la nécessité de produire un plaisir immédiat ne doit pas être considérée une dégradation de l'art du Poète » (Wordsworth, 1974, 1: 140).

Dans la Préface de 1802, le poète n'est pas encore défini, comme il le sera plus tad, comme un modèle absolu qui conduit le lecteur. Mais est déjà écartée la conception classique de l'art selon laquelle le lecteur est un sujet passif qui se borne à prendre plaisir dans ce qui lui est donné : la critique des œuvres populaires qui ne visent qu'à plaire au public se trouve déjà dans le texte de 1802. Pour Wordsworth, dans cette phase de sa carrière, poète et lecteur se joignent en ce que celui-là exprime des sentiments spontanés que celui-ci doit aussi éprouver tout en résonant avec les sentiments originaux du poète. Selon les idées alors développées par le mouvement romantique (cf. Woodmansee, 1994), le poète doit stimuler un principe de lecture active, que l'on peut appeler herméneutique : «je dois demander à mon lecteur qu'au moment de juger ces poèmes, il décide authentiquement à travers ses propres sentiments et non pas en réfléchissant sur ce qui est probablement le jugement d'autrui » (Wordsworth, 1974, 1: 156). Le poète produit une œuvre originale qui décrit des sentiments spontanés libérés de toute imitation et c'est ce modèle même que le lecteur doit assumer, cherchant lui aussi à éprouver des sentiments authentiques et à faire ainsi une lecture originale : il doit imiter le poète dans la recherche de l'originalité. En bref, bien que la Préface présente déjà pas mal de traits de l'idéologie romantique sur la création, on n'y trouve pas encore l'expression complète de l'idée d'indifférence pour les lecteurs qui forment l'audience, et la relevance du jugement que ceux-ci puissent former sur l'œuvre du poète n'y est pas niée non plus.

C'est l'indifférence face à l'audience et à son jugement qui sera affirmée catégoriquement dans «Essay, Suplementary to Preface» de 1815, un autre texte important pour la théorisation de l'esthétique romantique et qui a servi d'introduction à une nouvelle édition des Ballades Lyriques. La transition de la Préface de 1802 vers l'Essai représente le passage de l'idéal d'une audience en résonance avec les sentiments originaux du poète à la conception selon laquelle le vrai poète est forcément en opposition à l'audience (cf. Franta, 2007: 68). On peut également décrire ce passage selon les deux formes de l'imitation décrites dans l'œuvre de René Girard. D'une part, l'imitation positive que nous caractériserons pour le moment comme l'existence d'une appropriation non rivale du texte, traduite dans une empathie et une identité entre poète et lecteur, une appropriation dans laquelle celui-ci assume un principe de lecture active, peut-être même en récréant le texte. Plus loin on verra de façon plus détaillée que l'imitation positive est une imitation non rivale qui assure la transmission et la transformation de la culture. Proche de la tradition classique de la mimesis en art, cette forme d'imitation informait encore le texte de 1802. Par contre, celui de 1815 se fonde sur l'imitation negative qui consiste dans l'affirmation de la différence, de l'originalité absolue et dans le refus de l'imitation. Il se fonde sur l'imitation négative basée dans la rivalité, laquelle est, on le verra, aussi dépendante des autres que l'imitation positive. Il s'agit là d'un texte qui a contribué à la diffusion de ce que Girard a appelé «le mensonge romantique » (Girard, 1961). 
5 L'Essai est entièrement axé sur les thèmes de la renommée et de la réception des œuvres littéraires. L'argument central consiste à dire que les grands écrivains du passé n'ont pas été reconnus en tant que tels de leur vivant. Bien que les exemples présentés soient historiquement inexacts, Wordsworth soutient que des auteurs comme shakespeare et Milton ont été ignorés par le public au profit d'autres auteurs que la postérité a par ailleurs oubliés.

Who is there that now reads the 'Creation' of Dubartas? Yet all Europe once resounded with his praise; he was caressed by kings; and, when his Poem was translated into our language, the Faery Queen faded before it. (...) it is certain that these Poems of Milton are now much read, and loudly praised, yet were they little heard of till more than 150 years after their publication (Wordsworth, 1974, 3: 67; 70).

6 Shakespeare lui-même a dû adapter ses pièces au goût de l'audience pour gagner un peu de reconnaissance, et il aurait sûrement été vaincu par un concurrent de moindre talent, s'il avait participé à un concours littéraire (Ibid.). Ce qui veut dire que la popularité d'un auteur de son vivant n'est pas un critère qui permette de juger de la qualité originale d'une œuvre: "arrêtez la répétition insensée du mot populaire comme s'il n'y avait aucune épreuve d'excellence dans celui qui est le premier des arts » (Wordsworth, 1974, $3: 83)$.

7 Dans l'Essai apparait donc l'idée de l'artiste génial, original, « celui qui introduit un nouveau élément dans l'univers intellectuel » (Wordsworth, 1974, 3 : 82), qui ne sera pas estimé à sa juste valeur de son vivant. La réception de l'œuvre dans l'indifférence signale sa qualité ! Aussi le poète doit-il créer dans le public la sensibilité qui reconnaitra sa génialité à long terme : "n'importe quel auteur, dans la mesure où il est grand et en même temps original, a dû créer le goût par lequel il a à être apprécié ; il en fut ainsi et il en sera toujours ainsi » (Wordsworth, 1974, $3: 80$ ).

8 L'idée selon laquelle la vraie originalité n'est pas reconnue au temps de son créateur était elle-même une idée originale à l'époque, rare avant la fin du XVIII ${ }^{\mathrm{e}}$ siècle. Elle exempte l'artiste aux critiques du public et seul son auto-critique et son cercle d'amis pourront éventuellement lui servir de guide (Kristeller, 1983 : 107). L'art et l'artiste entrent dans un processus d'imitation négative en déclarant l'indifférence au jugement $\mathrm{du}$ public. L'autonomie et l'auto-suffisance de l'artiste se caractérisent précisément par cette indifférence et c'est cela sa différence. Au contraire, l'audience est conduite par l'esprit d'imitation: les œuvres que chacun apprécie sont les œuvres populaires que la foule aime. Par conséquent, une œuvre originale, qui ne participe pas de l'imitation, est condamnée à l'incompréhension.

Dans sa reconstruction fictionnée de la réception historique des œuvres littéraires, Wordsworth réfère fréquemment les cas de Shakespeare et Milton. Mais on peut deviner que l'Essai a en vue un autre exemple, celui de l'auteur même, William Wordsworth. Le poète romantique était persuadé que son œuvre ne serait reconnue qu'à long terme :

sketch of my own notion of the constitution of Fame has been given; and, as far as concerns myself, I have cause to be satisfied. The love, the admiration, the indifference, the slight, the aversion, and even the contempt, with which these Poems have been received, knowing, as I do, the source within my own (...) that the products of my industry will endure (Wordsworth, 1974, 3: 80).

10 Que s'est-il passé pendant la période qui s'étend depuis la publication de la seconde édition des Ballades Lyriques jusqu'à celle de l'Essai ? Après une phase initiale d'une certaine réussite, les poèmes de Wordsworth deviennent la cible de la critique et peinent 
à se vendre. Ce sont là les faits que l'on avance normalement pour expliquer l'évolution de la pensée du poète entre la publication de la Préface et celle de l'Essai. Ces faits expliquent que le poète affirme ne plus chercher la communion avec le public - la position soutenue en 1802 - et lui communique son indifférence face à la critique et au marché. Ce sont donc des raisons existentielles qui expliquent la nouvelle conception de l'art développée dans l'Essai. Elles ont souterrainement engendré la formulation romantique de Wordsworth sur la "génialité » et l' «originalité ", de «l'artiste incompris ", etc, qui dès lors sont devenus des lieux-communs de la conception moderne de l'art.

11 On répète souvent que les critiques à l'oeuvre de Wordsworth ont été normalement négatives pendant longtemps (cf. Simonsen, 2005, pour une synthèse). Celles de Francis Jeffrey, le critique littáraire anglais le plus proéminent de la période romantique, ont été particulièrement importantes. En 1807 il écrit une critique dévastatrice des Poems, In Two Volumes, de Wordsworth. À une époque où la littérature jouissait déjà d'une grande circulation, le rôle des critiques littéraires était aussi déjà très important et le prestige de quelqu'un comme Jeffrey surpassait de beaucoup celui de Wordsworth (Hesse, 2005 : 174). Son avis n'a pas fait augmenter la consommation des Poems dont le niveau de vente est resté toujours très bas. En réponse à cette faible réception et pour antéciper des critiques comme celles de Jeffrey, Wordsworth a écrit en 1807 à Lady Beamount une lettre « remarquable» dans l'expression des auteurs de l'édition critique des œuvres en prose de Wordsworth (Wordsworth, 1974, $3: 56$ ). Il y a un passage sur les raisons de son manque de popularité qui sera essentiellement répété dans l'Essai de 1815, tout comme il a été cité plus haut.

That every great and original writer, in proportion as he is great or original, must himself create the taste by which he is to be relished; he must teach the art by which he is to be seen; (...); but for those who dip into books in order to give an opinion of them, or talk about them to make up an opinion - for this multitude of unhappy, and misguided, and misguiding beings, an entire regeneration must be produced; and if this be possible, it must be a work of time (Wordsworth, 1969, 2: 150).

12 Cet extrait ne constitue pas la conclusion d'un argument théorique abstrait. La lettre, «remarquable», est une invective permanente de Wordsworth contre les critiques et la non reconnaissance dont ses poèmes sont l'objet. Juste avant le passage cité, les critiques sont décrits comme des «juges incompetents. Ce sont des gens qui, dans l'agitation de leur vie oisive, ne lisent pas des livres et se bornent à y jeter un coup d'oeil pour pouvoir en parler " (Ibid.). À cela le poète répond avec son indifférence : " pour conclure, mes oreilles sont sourdes à ce bourdonnement oisif, et ma chair est insensible comme l'acier à ces petites piqûres » (Ibid.).

13 La lettre manifeste la méfiance de Wordsworth envers l'audience qu'il avait auparavant voulu captiver dans la Préface de 1802. En essayant de surmonter cette situation, l'auteur des Ballades Lyriques a gagé que ses poèmes seraient reconnus dans la postérité. On pourrait dire que le gage s'est bel et bien réalisé dans la mesure où Wordsworth a fini au panthéon de la poésie. Mais cela ne doit pas nous voiler la grande audace de ses jugements sur sa postérité, qui vont à l'encontre de l'avis géneral de son époque et ne se fondent que dans une analyse volontariste et introspective du poète sur lui-même (Hesse, 2005 : 175). L'auteur romantique affirme que, parce qu'ils sont originaux et différents, ses poèmes ne sont pas reconnus, quand, à vrai dire, c'est le fait qu'ils ne sont pas reconnus qui mène Wordsworth à insister sur leur originalité. Sûrement une telle insistance 
n'existerait pas si la reconnaissance existait ! La déclaration performative de l'originalité est une réaction à l'incompréhension. C'est le désir de reconnaissance, l'inexistence de reconnaissance, qui a produit le discours théorique sur l'originalité et sur l'incompréhension dans le court terme à laquelle est condamné le vrai artiste.

En 1814, Jeffrey a analisé une autre oeuvre de Wordsworth, The Excursion, en publiant une critique encore plus dévastatrice («this will never do»). Les éditeurs des Oeuvres Complètes en Prose affirment que le critique romantique est devenu «un scandale ('stumbling-block') pour Wordsworth ", et que l'Essai est produit en tant que réponse directe aux critiques, notamment à Jeffrey (Wordsworth, 1974, $3: 59$ ). Dans le langage de la théorie mimétique de René Girard, Jeffrey est devenu le modèle-obstacle de Wordsworth: haï mais simultanément objet d'une permanente attention toujours en attente d'un signe favorable. Dans le même langage, Wordsworth était animé du ressentiment typique de l'homme moderne du souterrain et, en effet, pour plusieurs critiques de l'époque, l'Essai constituait une attaque personnelle à Jeffrey et les écrits de Wordsworth « révélaient le ressentiment et la douleur qu'il avait souffert» (Wordsworth, 1974, $3: 60$ ). Le texte de 1815 a été une façon pour Wordsworth de se proteger des jugements négatifs de la critique et du public, les déclarations sur l'indifférence à la critique et l'autonomie et la différence du poète naissant de cette situation existentielle. Ces déclarations sont en réalité inséparables de son attention extrême aux jugements d'autrui (cf. Hesse, 2005 : 174). Tout en critiquant le critère de la popularité comme critère de valorisation des oeuvres, l'Essai révèle une obsession envers la popularité (Murphy, 1993: 196). Face à l'impopularité, la meilleure stratégie est de déclasser l'importance de la popularité. Face à l'indifférence des autres, la meilleure stratégie est d'affirmer sa différence. Face à l'extrême dépendance du jugement des autres, la meilleure stratégie est de s'affirmer indépendant de cette dépendance. Le poète est secrètement lié aux autres mais affirme publiquement l'inexistence de cette liaison. Le fait notable, mais parfaitement compréhensible, c'est que cette stratégie existentielle, mimétique, a produit le discours de l'Essai, lequel est devenu lieu-commun sur « artiste ", « original», «créatif», « génial», « incompris». Elle a produit le discours de l'artiste original qui n'est pas reconnu de son vivant.

\section{Wordsworth et la loi du copyright}

Les idées développées dans l'Essai, notamment l'idée que l'auteur vraiment original ne verra pas sa valeur reconnue, alors que les oeuvres de qualité médiocre obtiennent une reconnaissance populaire immédiate, sont importantes dans la mesure où elles ont formé aussi la pensée de l'auteur des Ballades Lyriques sur la question du copyright. Il s'est toujours beaucoup intéressé à ce type de législation qui, au début du XIX ${ }^{\mathrm{e}}$ siècle, tournait autour des périodes de protection (d'exclusivité d'impression) qui devaient être accordées aux livres. En Angleterre, jusqu'à 1814, la période de protection était celle établie par la première loi du copyright, le Statute of Anne (1710) qui stipulait 14 années pour les nouveaux livres et 21 pour les livres déjà imprimés. En 1808, Wordsworth écrivait que non seulement ces délais étaient trop courts mais aussi qu'une nouvelle loi, alors en discussion et qui prévoyait des périodes de 28 ans, était encore insuffisante, une protection plus longue étant nécessaire. La période de 28 ans était insuffisante pour " établir la réputation des œuvres originales ", ce qui était justifié de la façon suivante : 
I am told that it is proposed to extend the right from 14 years, as it now stands, after the decease of authors, till 28, this I think far too short a period; at least I am sure that it requires much more than that length of time to establish the reputation of original productions, both in Philosophy and Poetry, and to bring them consequently into such circulation that the authors, in the Persons of their Heirs or posterity, can in any degree be benefited, I mean in a pecuniary point of view, for the trouble they must have taken to produce the works. The law as it now stands merely consults the interest of the useful drudges in Literature, or of flimsy and shallow writers, whose works are upon a level with the taste and knowledge of the age; while men of real power, who go before their age, are deprived of all hope of their families being benefited by their exertions (Wordsworth, 1969: 266-7).

L'argument consiste à dire que les périodes de protection courtes ne sont bénéfiques que pour les œuvres populaires qui sont immédiatement acceptées. Quant aux œuvres vraiment originales, elles prennent bien longtemps à être reconnues. Et quand cela finalement arrive, elles sont déjà dans le domaine public, l'auteur se voyant privé de la juste rémunération à laquelle il avait droit. À remarquer que le point axial dans l'argumentation de Wordsworth sur le fondement du copyright réside toujours dans la distinction entre œuvres populaires - sans qualité - et œuvres vraiment originales dont la qualité prend longtemps à être reconnue et à devenir populaire.

En 1814 une nouvelle loi anglaise sur le copyright fixe effectivement une période de 28 ans après la publication et, au cas où l'auteur serait encore vivant au bout de cette période, la protection continuerait jusqu'à sa mort. Évidemment cette nouvelle loi ne satisfaisait pas Wordsworth qui soutenait la nécessité de périodes encore plus longues. Plus tard, en 1830, il revient au sujet tout en insistant qu'une durée courte "primait la médiocrité parce qu'elle poussait les auteurs à rechercher des effets immédiats » (Wordsworth, 1979 : 225). L'intérêt de Wordsworth pour la question du copyright s'est encore approfondi quand un de ses admirateurs et amis, le député Serjeant Talfourd, a proposé d'altérer la loi de 1814. Dans la proposition de Talfourd, la période de protection devrait comprendre la durée de vie de l'auteur plus 60 ans après sa mort. La proposition accueillait aussi l'argument selon lequel les périodes courtes stimulaient la recherche de la réussite immédiate ; or, la loi devrait avoir comme objectif de protéger les auteurs "originaux " en opposition aux auteurs " populaires ». Le parcours de la loi proposée par Talfourd a suivi de nombreuses péripéties au Parlement et ses versions initiales ont été refusées ${ }^{1}$. Dans le contexte du grand débat public suscité par la proposition de loi, Wordsworth a écrit plusieurs articles et lettres en sa faveur, aussi bien auprès des parlementaires que du public. Dans une lettre adressée à Talfourd (Wordsworth, 1974, $3: 313-4)$, il a développé l'argumentation classique selon laquelle la loi du copyright étant une common law, elle constitue le droit naturel de l'auteur à la propriété de son œuvre. Cette position se soutient d'une théorie du travail qui remonte à Locke, selon laquelle la propriété découle du travail de l'auteur pour produire l'œuvre. Depuis le XVII siècle jusqu'à aujourd'hui, cette position s'est toujours opposée à une autre qui considère le copyright un droit positif créé artificiellement pour encourager la création de nouvelles œuvres (cf. Machuco Rosa, 2015). Nous verrons plus loin que c'est cette seconde position qui a réellement influencé les lois dans les pays anglo-saxons.

Dans un autre texte, publié en 1838, Wordsworth a développé l'autre argument qui nous est déjà familier. Il réfère que l'objectif de la loi ne doit pas être de favoriser la production et la circulation de livres mais de «bons livres » et surtout de favoriser les auteurs qui regardent au-delà du court terme et s'adressent aux générations futures. La loi doit donc encourager: encourager la création d'œuvres qui ne sont pas populaires mais qui le 
deviendront dans l'avenir; encourager la création d'œuvres vraiment originales. Soulignons de nouveau que, dans cette justification du copyright, celui-ci n'est pas une loi destinée à encourager la création tout court, mais plutôt la création de "bonnes » œuvres, celles dont la reconnaissance exige beaucoup de temps. D'où la nécessité d'élargir le plus possible les périodes de protection, ce qui est clairement exprimé dans le passage suivant :

If certainties and probabilities be looked at with more discernment than is shown by these petitioners, it will be found that a book for which there is a great demand would he sure of being supplied to the public under any circumstances; but a good book for which there might be a continued demand, though not a large one, would be much more sure of not becoming a "dead letter," if the proposed law were enacted than if it were not. It is well known among the intelligent that the non existence of copyright for English authors in America is a great hindrance to the republication of standard works (Wordsworth, 1974, 3: 309).

19 Les œuvres populaires n'ont pas besoin de protection spéciale, vu qu'elles ont a priori un marché assuré. La loi du copyright doit viser à promouvoir la création et la protection d'œuvres originales qui, en absence d'une protection à long terme, resteront «lettre morte ». La loi du copyright doit protéger l'artiste original et indépendant qui, en échange des idées et formes nouvelles qu'il transmet à la société, doit recevoir de la part de celleci une protection qui lui assure ainsi qu'à ses descendants une récompense financière. Pourtant, l'auteur romantique ne crée pas dans la recherche du profit immédiat, mais dans "l'espoir d'être toujours bénéfique pour l'humanité ", comme Wordsworth l'a écrit au premier-ministre anglais, Robert Peel, en 1835 (Wordsworth', 1979 : 21). En échange, le gouvernement doit protéger l'artiste et ses descendants.

Cette argumentation de Wordsworth ne sous-tend pas, au moins dans l'essentiel, les lois modernes du copyright, comme on va voir tout de suite. En effet, le projet initial de Talfourd a été refusé. Une nouvelle loi - qui ne fait aucune allusion aux œuvres qui ne sont reconnues que dans un avenir indéterminé - a fini néanmoins par être approuvée en 1842, établissant pour la première fois dans la loi anglaise un copyright post-mortem. La période du copyright est devenue soit la durée de vie de l'auteur plus 7 ans après sa mort, soit 42 ans après la publication, la période la plus longue validant le choix. Wordsworth a reçu cette nouvelle loi avec une satisfaction modérée, car pour lui le copyright devrait être perpétuel (Wordsworth, 1974, 3 : 313). On peut effectivement affirmer que la loi de 1842 n'a pas accueilli l'opinion de l'auteur romantique (Seville, 2003 : 215).

Comment évaluer de façon plus précise les idées de Wordsworth à la lumière de la philosophie et du contenu des lois modernes du copyright et du droit d'auteur ? Comme on l'a vu, ces idées étaient centrées sur l'affirmation performative de l'artiste vrai comme étant celui qui est original, et vu que les œuvres originales exigent longtemps pour être reconnues, la durée du copyright doit être longue. Le copyright aurait donc comme fondement l'encouragement de la création et de la protection des œuvres originales, de qualité, et non pas des œuvres immédiatement populaires.

En mettant momentanément de côté la question des périodes de protection, on avancera que le discours sur l'« originalité » est une des bases essentielles des lois du copyright et du droit d'auteur. Plusieurs auteurs (Jaszi, 1991; Rose, 1993; Woodmansee, 1994) ont soutenu que les idées romantiques ont contribué décisivement à l'émergence des notions modernes d' " auteur ", " créateur ", " original ", lesquelles ont fini par être consacrées dans la loi. Cette contribution, qui a commencé au milieu du XVIII ${ }^{\mathrm{e}}$ siècle avec des écrivains comme Edward Young, n'est pas dûe spécifiquement à Wordsworth, mais à la 
généralité du mouvement romantique. Et, en effet, les différentes législations nationales se sont progressivement centrées sur les "œuvres originales", les "créations intellectuelles » des « auteurs $»^{2}$. Ceci constitue une nouveauté historique et il est certain que les idées romantiques ont contribué à l'émergence de ce type de langage juridique. En outre, il y a eu tout au long des siècles des décisions de tribunaux qui ont interprété l'exigence d'originalité de façon que nous dirons substantive : les juges déterminaient si les œuvres atteignaient ou non un certain standard de suffisante originalité (cf. Bracha, 2008 ; Casas Vallés, 2009).

Toutefois, l'interprétation de l'originalité en tant qu'expression de la qualité substantive de l'oeuvre a été bien moins la règle que l'exception. Une interprétation des lois du copyright et du droit d'auteur en tant qu'expression de l'idéal romantique de la créativité escamote le fait que ces lois présentaient l'exigence d'originalité complètement séparée des notions de nouveauté ou de mérite. Si l'originalité au sens romantique est devenue un fondement des lois du copyright, les textes mêmes des lois ont finalement très peu à voir avec l' idéologie romantique (Braha, 2008: 209). Par exemple, le Code du Droit d'Auteur portugais protège « les créations intellectuelles ", indépendamment de leur mérite, mode de communication et objectif (Article $2^{\mathrm{e}}$ ). Dans la plupart des lois actuellement existentes, les exigences pour qu'une œuvre jouisse de protection et, par conséquent, soit légalement " originale ", sont absolument minimes; aucun besoin aujourd'hui de formalités - comme l'enregistrement - pour que l'œuvre soit protégée par le copyright et le droit d'auteur. Le droit est attribué automatiquement. La seule condition est surtout que l'oeuvre ne soit pas littéralement une copie d'une autre, indépendamment de ses mérites. Le copyright se fonde dans l'idée d'originalité et créativité, mais les exigences d'originalité sont minimes (Bracha, 2008). On peut donc en conclure que, si d'une part le mythe de l'originalité romantique se trouve présent dans le fondement, c'est-à-dire dans le discours qui légitime l'existence de protection intellectuelle, de l'autre l'articulation concrète de la loi - et la pratique des tribunaux - a très peu de rapport avec ce mythe légitimateur.

La justification et la détermination des périodes de protection des œuvres n'a quasiment rien à voir avec les arguments spécifiques avancés par Wordsworth. Bien que l'idée d'originalité soit un mythe fondateur, on a vu que l'exigence d'originalité substantive n'existe pratiquement pas. Complètement absente de l'esprit et de la lettre de la loi se trouve aussi l'idée qu'elle encouragerait la création d'oeuvres pas populaires qui ne seraient reconnues qu'à long terme. Le fait que les périodes de protection sont de plus en plus longues ne se justifie pas de ce type d'argument. Les lois actuelles de protection intellectuelle ont surtout comme point de départ de leur fondement la nature non-rivale (ma consommation ou usage de l'oeuvre ne fait diminuer en rien sa quantité disponible) et non-exclusive (la publication d'une oeuvre est un processus irréversible au sens où il est très difficile de rendre non public ce qui a déjà été public) de l'information. La question qui se pose par la suite est de trouver la justification économique pour l'existence de lois de protection intellectuelle d'information, lesquelles rendent l'information rivale et exclusive (cf. Landes \& Posner, 2003 ; Lévêque \& Menière, 2003). Ces lois ont une inspiration utilitariste, leur objectif étant de maximiser le bien-être social, ce qui se traduit dans la recherche d'un équilibre entre l'efficacité statique et efficacité dynamique de l'information. Si l'information n'était pas protégée, l'efficacité statique serait aujourd'hui maximale, car tout le monde y aurait accès à bas coûts. Mais cela aurait pu impliquer que les auteurs n'auraient aucune motivation à créer et, par conséquent, la protection existe pour motiver les auteurs à créer dans le futur. Donc, la loi 
doit chercher un équilibre entre l'efficacité statique qui se perd à cause de la protection, et l'efficacité dynamique qui se gagne (assurer la création dans l'avenir). Elle prétend être un encouragement à la création de nouvelles oeuvres, mais, différemment de la théorie des encouragements de Wordsworth, elle est indépendante du mérite substantif de ces œuvres.

On ne prétend pas que les lois actuelles ont réussi à atteindre le point d'équilibre référé qu'il est très difficle, voire impossible, de déterminer exactement. Le conflit ou la tension entre, d'une part, les dispositions visant à garantir une imitation positive fondée dans la non-rivalité et, d'autre part, celles qui excluent (partiellement) l'oeuvre de l'usage public, est permanente. En voici deux exemples.

Nous avons fait référence plus haut les actions de Wordsworth voulant influencer le Parlement anglais à approuver une loi qui prolonge les périodes de protection. Ce type d'action indique que les raisons qui mènent à élargir les périodes ont peu à voir avec l'idée de "création originale ", et surtout avec l'idée selon laquelle les périodes doivent être longues parce que les créations originales demandent longtemps à être acceptées et valorisées par le public. Comme nous l'avons dit aussi, l'extension prévue par la loi de 1842 n'a eu rien à voir avec les idées générales de Wordsworth sur l'originalité, mais elle a certainement découlé en partie du lobbying qui, outre Wordsworth, des auteurs comme Carlyle, Arnold et Coleridge ont conduit, tous bien conscients des gains financiers personnels permis par les périodes longues (cf. Seville, 2003 : 30). À vrai dire, les périodes ont été successivement prolongées. Par exemple, en Angleterre on a commencé à 14 ans stipulés par le Statute of Anne et en 1842 on a passé à la période de durée de vie de l'auteur plus 7 ans ou alors un total de 42 ans. Plus tard, cette période s'est élargie à 50 ans après la mort de l'auteur. En France et au Portugal il y a eu un mouvement semblable avec le passage de la période de la durée de vie de l'auteur plus 5 ans (en France, en 1793) à la période de 30 ans plus tard. Au Portugal, en 1851, la période s'est fixée en 50 ans. Plus récemment dans les deux pays la période a été élargie à 70 ans. Ces extensions n'ont pas de justification dans les idées romantiques. La raison réelle est le lobbying de défense des intérêts personnels, normalement justifié par la théorie du travail qui affirme que si c'est le travail personnel qui a créé l'œuvre d'art, alors celle-ci est la propriété de son auteur, tout comme Wordsworth l'a également soutenu. L'augmentation des périodes de protection implique une entrave à la circulation de l'information et contrarie très souvent l'idée d'encouragement à la création.

Le second exemple va dans le sens opposé. Bien que la loi attribue un monopole à l'auteur ou à ses représentants dans l'exploitation et l'accès à l'œuvre, il y en a néanmoins des exceptions. Ces exceptions permettent, certes de façon très limitée, l'accès public libre aux oeuvres et à leur partage non rival, ce qui ouvre la voie à quelques formes d'imitation positive. La raison en est que les lois du copyright et du droit d'auteur ne protègent qu'une certaine expression concrète mais ne protègent jamais les idées elles-mêmes. En outre, les exceptions à l'exclusivité se traduisent dans ce qu'on appelle l'usage loyal. Par exemple, dans le cas du Code portugais, l' Article $75 .^{\circ} \S 2$ dit que sont licites sans le consentement de l'auteur la reproduction à des fins exclusivement privées et sans objectifs commerciaux, la reproduction et la distribution à des fins d'enseignement et d'éducation, la reproduction en bibliothèques, etc. Des règles semblables existent dans les pays anglosaxons, notamment les règles de fair use prévues dans l'Article 17. ${ }^{\circ} \$ 107$ du Code des États Unis, comme le commentaire, la critique ou la parodie et l'usage pour la recherche. Quoique de façon limitée, ces règles se fondent dans la non rivalité de l'information et 
assurent des formes d'imitation positive. C'est le rapport entre ce type d'imitation et la propriété intellectuelle que nous allons maintenant analyser.

\section{Imitation positive et propriété intellectuelle}

Même si l'existence de l'usage loyal assure un certain accès libre aux œuvres, il s'agit d'un accès très limité parce que la loi est de plus en plus orientée dans le sens d'assurer les droits d'exclusivité. Qu'elle se justifie d'une théorie du travail ou d'une théorie utilitariste sur les encouragements à la création future, la loi est axée sur l'auteur, sur sa création individuelle et sur le contrôle presque total de l'accès à l'œuvre et de son usage par des tiers. Mais serait-t-il possible d'encadrer dans une perspective girardienne une autre façon de concevoir la création? Dans cette perspective, quelles seraient les modalités légales d'accès et d'usage des œuvres au-delà de celles prévues dans les lois du copyright et du droit d'auteur? Ce nouveau point de vue doit avoir comme fondement l'idée que la création, l'accès et l'usage de l'information doivent «maximiser» l'imitation positive. L'imitation positive est l'imitation non rivale. En absence d'une protection légale comme celle assurée par le copyright et par le droit d'auteur, l'information est précisément un bien non rival qui peut être librement utilisé par n'importe quel individu, sans que cet usage heurte un usage identique par d'autres individus. L'imitation positive comme fondement d'accès et usage de l'information ne relève pas d'une quelconque théorie de l'encouragement à la création, y compris celle des motivations économiques qui inviteraient les créateurs à créer.

Plus spécifiquement, l'imitation positive peut être fondée dans l'expérience de recevoir gratuitement, expérience qui a lieu chaque fois que des êtres humains reçoivent gratuitement (gratuitous) et distribuent ensuite aux autres ce qu'ils ont reçu librement ( freely) et sans calcul (Steinmair-Pösel, 2014: 229). Ainsi s'est déroulé le processus millénaire de transmission culturelle et ainsi s'opère la plupart de l'apprentissage. Jusqu'à l'émergence des premières lois du copyright, au XVIII ${ }^{e}$ siècle, la création, transformation et distribution de l'art et du savoir se faisait sous la modalité de l'imitation positive, c'est-àdire librement. Par exemple, au Moyen Âge, la copie et la modification des œuvres de l'Antiquité faisait partie de la création et diffusion intellectuelles. Les œuvres étaient perçues comme des entreprises collectives qui réorganisaient des éléments des œuvres anciennes en ajoutant quelque chose de nouveau'. L'absence alors de droits légaux d'auteur ou de copyright et de récompense financière n'a pas empêché la production d'œuvres littéraires, fait qui constitue un argument qui met en question la justification du copyright. Un point important réside dans le fait que les théologiens du Moyen Âge ont élaboré un corps doctrinaire qui concevait la connaissance comme une modalité d'imitation positive, telle que celle-ci peut être définie par le célèbre passage de Saint Matthieu où Jésus dit : " vous avez reçu gratuitement, donnez gratuitement » (Mt. 10, 8). On a ainsi établi que la connaissance est une grâce de Dieu; c'est un don qui, sous peine de péché, ne peut pas être vendu (Hesse, 2002).

Il s'agit de considérer l'imitation positive en conjonction avec une conception non romantique de l'activité créative - une conception qui souligne l'importance de la reconfiguration de quelque chose qui pré-existe pour qu'il y ait une authentique activité créative - en tant que fondement de l'existence (ou non) de mécanismes de protection intellectuelle. ${ }^{4}$ Nous soulignons que cette fondamentation n'est ni celle de la théorie du travail ni celle de la théorie utilitariste. C'est plutôt une théorie de la planification sociale, 
une théorie sémiotique, qui assure une culture attirante, vivante, qui permette le maximum d'accès à l'information, qui crée les conditions pour que tout le monde accède aux modèles qui existent et puisse créer à partir d'eux, en participant ainsi dans la création des significations culturelles dans le cadre de la formation d'une tradition qui contribue à l'existence de "communautés de mémoire » (cf. Fisher, 2001). Comment l'imitation positive peut-elle être au fondement d'une telle culture sémiotique participative, étant donné que l'effet de nombreuses dispositions des lois de la propriété intellectuelle est précisément de l'éviter?

31 Ceci est possible grâce aux nouvelles formes de "propriété » intellectuelle qui permettent de dépasser l'antinomie apparemment insurmontable entre imitation positive et protection légale de l'information. Le meilleur exemple est la licence d'utilisation General Public Licence (GPL). Elle apparait dans le contexte de l'informatique des années 1980, quand les producteurs de software se sont mis à rivaliser intensément, en produisant des incompatibilités entre les logiciels et en protégeant le code-source des programmes par le copyright; autrement dit, ils ont déclenché une dynamique mimétique de réciprocité négative dans l'espoir d'atteindre une position monopoliste, mais ne faisant en fait que morceller le système de programmes qui aurait permis l'inter-opérabilité entre les différents types d'ordinnateurs. C'est cette situation qui est à l'origine de la conception par Richard Stalmann (cf. Stalmann, 2001) d'un mécanisme légal destiné à forcer la coopération et à cesser la compétition négative qui affectait la production de software à l'époque. Ce mécanisme est une licence dotée de valeur légale. C'est la licence GPL qui constitue un artifice visant à restaurer un régime d'imitation positive dans le domaine de l'information. La licence se fonde sur certaines libertés (« freedoms ») dans l'utilisation de l'information : (1) la liberté de copier, (2) la liberté de modifier et (3) la liberté de publier les modifications introduites. Mais la clause fondamentale de la licence GPL consiste à stipuler que l'information modifiée doit être soumise aux conditions que l'on vient d'énoncer dans les trois libertées préalables. Cela veut dire qu'il faut une apparente réduction des libertés (1), (2) et (3) pour que l'information continue libre. Dans le cas du software, cela signifie que le code-source ne peut pas être fermé et doit rester publiquement disponible et modifiable. Comme Stalmann insiste, « libre » n'a rien à voir avec «à l'oeil » mais tout à voir avec l'obligation de donner.

La clause distinctive de GPL consiste à assurer une certaine liberté à travers une apparente restriction à la liberté tout en assurant que les libertés (1), (2) et (3) ne peuvent pas disparaître ; aussi implique-t-elle une plus grande liberté. La clause (4) est virale, elle assure que la liberté se propage, qu'elle ne peut pas être annulée. Elle garantit que la liberté se propage. Un artifice comme GPL restaure l'état originaire de non-rivalité et d'appropriabilité publique - sa nature intrinsèquement coopérative - de l'information. Les œuvres crées et distribuées sous GPL sont la conséquence de l'imitation positive que la licence même établit comme sa condition fondamentale. Elle fonde un lien social basé dans une structure triadique, car un individu $B$ qui reçoit de $A$ doit redistribuer à $C$, ce qui engendre le cycle $\mathrm{A} \rightarrow \mathrm{B} \rightarrow \mathrm{C} \rightarrow \mathrm{D} \rightarrow \ldots . \rightarrow \mathrm{A}$. Il s'agit encore une fois de mettre en place légalement l'imitation positive entendue comme l'expérience de redistribuer ce que l'on a reçu gratuitement.

33 Au début, la licence GPL a été conçue pour le cas spécifique du software. Aujourd'hui elle encadre légalement l'accès à un numéro immense de programmes. Toutefois il est possible d'utiliser la philosophie - et les termes mêmes - de GPL dans des licences à appliquer aux différentes créations artistiques en général, assurant ainsi l'existence de 
formes spécifiques de protection intellectuelle fondées sur l'imitation positive. C'est le cas des licences Creative Commons. Elles s'inspirent de la Genenal Public Licence originale, mais elles sont flexibles au sens où elles ne forcent pas un individu à assurer à d'autres individus la possibilité de modifier l'information qu'il a lui-même modifiée. Elles visent aussi à permettre que l'auteur garde ses droits d'attribution et que l'œuvre garde son intégrité et elles n'empêchent pas l'exploitation commerciale des œuvres. Le tableau suivant énumère les différents types de licences Creative Commons.

\begin{tabular}{|c|c|c|c|}
\hline Licences & Attribution & Intégrité & $\begin{array}{l}\text { Partage } \\
\text { obligatoire }\end{array}$ \\
\hline Attribution- CC BY & Oui & Non & Non \\
\hline $\begin{array}{l}\text { Attribution - Partage dans les termes de } \\
\text { la même licence CC BY-SA }\end{array}$ & Oui & Non & Oui \\
\hline $\begin{array}{l}\text { Attribution - Interdiction de réalisation } \\
\text { d'œuvres } \\
\text { CC BY-ND }\end{array}$ & Oui & Oui & Non \\
\hline $\begin{array}{l}\text { Attribution - Usage Non -Commercial } \\
\text { CC BY-NC }\end{array}$ & Oui & Non & Non \\
\hline $\begin{array}{l}\text { Attribution - Usage Non-Commercial - } \\
\text { Partage dans les termes de la même } \\
\text { licence CC BY-NC-SA }\end{array}$ & Oui & Non & Oui \\
\hline $\begin{array}{l}\text { Attribution - Usage Non-Commercial - } \\
\text { Interdiction de réalisation d'œuvres } \\
\text { Dérivées CC BY-NC-ND }\end{array}$ & Oui & Oui & Non \\
\hline $\begin{array}{l}\text { Dédication Universelle au Domaine } \\
\text { Public CC0 }\end{array}$ & $\begin{array}{l}\text { Renonce à tous les droits } \\
\text { conférés par la loi du } \\
\text { copyright }\end{array}$ & & Non \\
\hline
\end{tabular}

Il y a aujourd'hui des millions d'œuvres licenciées dans les termes de Creative Commons. Le cas de Wikipedia, licenciée dans les termes de CC BY-SA, est particulièrement important. C'est un excellent exemple d'un projet collaboratif ouvert de production de contenus basée sur l'imitation positive. Mais si on préfère un régime intégral d'imitation positive - la gratuité absolue sans aucun devoir de réciprocité -, alors on utilisera la licence $\mathrm{CCO}$, renonçant à tous les droits de protection intellectuelle. 


\section{BIBLIOGRAPHIE}

ABRAMS, Meyer (1953). The Mirror and the Lamp: Romantic Theory and the Critical Tradition. Oxford: Oxford University Press.

BRACHA, Oren (2008). « The Ideology of Authorship Revisited: Authors, Markets, and Liberal Values in Early American Copyright. The requirement of originality ", Yale L. J., 118, pp. 186-271. CASAS VALLÉs, Ramon (2009). " The requirement of originality », in Estelle Derclaye (orgs.). Research Handbook on the Future of EU Copyright. Edward Elgar : Cheltenham, pp. 102-132. FISCHER, William (2001). « Theories of intellectual property », in Stephen Munzer (org.). New Essays in the Legal and Political Theory of Property. Cambridge: Cambridge University Press, pp. 168-199. FRANTA, Andrew (2007). Romanticism and the rise of the mass public. Cambridge: Cambridge University Press. GIRARD, René (1990). «Innovation and Repetition », SubStance, vol. 19, No. 2/3, pp. 7-20. GIRARD, René (1961). Mensonge Romantique et vérité Romanesque. Paris: Grasset. HESSE, Carla (2002). « The rise of intellectual property, 700 b.c.- a.d.2000: an idea in the balance », Dodalus, Spring, pp. 26-45.

HESSE, Scott (2005). Authoring the self-Self-Representation, authorship, and the print market in British poetry from Pope through Wordsworth. London: Routledge.

JASZI, Peter (1991). « Toward a Theory of Copyright: The Metamorphoses of 'Authorship'« Duke Law Journal, vol. 1991, pp. 455-502.

KRISTELLER, Paul (1983). « 'Creativity' and 'Tradition' « Journal of the History of Ideas, vol. 44, No. 1, pp. 105-113.

LANDES, William \& POSNER, Richard (2003). The Economic Structure of Intellectual Property Law. Cambridge : Harvard University Press.

LEVEQUE, François \& MENIERE, Yann. (2003). Économie de la propriété intellectuelle. Paris : La Découverte.

MACHUCO ROSA, António (2015). Do Copyright às Marcas Registadas - História e Fundamentos da Propriedade Intelectual. Lisboa: Chiado Editora.

MURPHY, Peter (1993). Poetry as an Occupation and an Art in Britain - 1760-1830. Cambridge:

Cambridge University Press.

ROSE, Mark (1993). Authors and owners: the invention of copyright. Harvard: Harvard University Press. SEVILLE, Catherine (2003). Literary copyright reform in early victorian England: The framing of the 1842 copyright act. Cambridge: Cambridge University Press.

SIMONSEN, Peter (2005). « The critical reception, 1807-1818 », in Andrew Bennett (org.). William Wordsworth in context. Cambridge: Cambridge University Press, pp. 54-60.

StALMAnN, Richard (2001). Free Software, Free Society: Selected essays of Richard M. Sallman. Boston: GNU Press. 
STEINMAIR-POSEL, Petra (2014). « Original Sin, Grace, and Positive Mimesis », in Vern Neufeld Redekop \& Thomas Ryba (orgs.). René Girard and Creative Mimesis. Lanham: Lexington Books, pp. 221-232.

WOODMANSEE, Martha (1994). The author, art and the market: Rereading the History of Aesthetics. New York: Columbia University.

WORDSWORTH, William (1969). The Letters of William and Dorothy Wordsworth, vol. 2 : The Middle Years : Part I : 1806-1811. Ernest De Selincourt \& Mary Moorman (orgs.) Oxford: Clarendon Press.

WORDSWORTH, William (1979). The Letters of William and Dorothy Wordsworth, vol. 5 : The Later Years : Part II : 1829-1834, Ernest De Selincourt (org.). Oxford: Clarendon Press.

WORDSWORTH, William (1974). The Prose Works of William Wordsworth, W. J. B. Owen \& Jane

Worthington Smyser ( orgs. ), 3 vol. . Oxford: Clarendon Press.

\section{NOTES}

1. Cf. Seville, 2003, pour une analyse détaillée des propositions de Talfourd et du parcours législatif qui a abouti à la loi anglaise du copyright de 1842 .

2. O Código do Direito de Autor portugais concerne les «créations intellectuelles » des " auteurs » (Article 1er), protégeant les « œuvres originales» (Article 2e).

3. Cf. Frosio, 2014, pour une analyse récente et détaillée de ce processus. Il écrit: «Thus in the medieval period, creativity was largely conceived as a participatory process, as opposed to the modern romantic individualistic perception » (356).

4. René Girard a explicitement développé cette conception dans Girard, 1990.

\section{RÉSUMÉS}

Cet article analise d'abord la façon dont quelques idées romantiques ont influencé les lois du copyright et du droit d'auteur et ensuite comment on peut concevoir d'autres régimes de propriété intellectuelle. L'article présente trois parties. La première partie concerne les raisons existentielles qui ont déterminé Wordsworth à formuler son idéologie romantique. La seconde examine les rapports de cette idéologie avec les lois de la propriété intellectuelle. La troisième présente de nouvelles formes de propriété intellectuelle comme les licences General Public Licence et Creative Commons pour montrer qu'elles sont en consonance avec la perspective girardienne de l'imitation positive.

Firstly the present paper examines the impact of some romantic ideas on copyright and authors' rights laws and then it proposes different regimes of intelectual property. The paper is divided into three parts. The first part is about the existential reasons that determined Wordsworth to formulate his romantic ideology. The second part examines the connections between this ideology and the laws of intelectual property. The third part features the new forms of intelectual property such as the General Public Licence and the Creative Commons in order to argue that they follow the Girardian perspective of positive imitation.

31 janvier 2018 
INDEX

Mots-clés : wordsworth, romantisme, copyright, Girard, imitation positive

Keywords : wordsworth, romanticism, copyright, Girard, positive imitation

\section{AUTEUR}

ANTONIO MACHUCO ROSA

Universidade do Porto

machuco.antonio[at]gmail.com 